\title{
BOUNDED EVALUATIONS FOR LINEAR ELLIPTIC DIFFERENTIAL OPERATORS ${ }^{1}$
}

\author{
THOMAS BAGBY AND JAMES E. BRENNAN
}

\begin{abstract}
Bounds for point evaluations are obtained for spaces of solutions of elliptic partial differential equations on generalized "Swiss cheese" compacta in Euclidean space. In this way we generalize recent approximation theorems for differential operators due to Polking.
\end{abstract}

1. Introduction and summary. We denote by $P(D)$ an elliptic partial differential operator of order $m$ with constant coefficients in $\boldsymbol{R}^{n}$; if $K \subset \boldsymbol{R}^{n}$ is compact, we wish to study the set $H(K)$ of functions which are defined on some open set containing $K$ and satisfy $P(D) u=0$ there. We let $1<p<\infty$ and $1 / p+1 / q=1$. The following is proved in recent work of Polking ([6], [7]).

THEOREM 1. The set $H(K)$ is dense in $L_{p}(K)$ for every compact nowhere dense set $K \subset \boldsymbol{R}^{n}$ of positive Lebesgue measure if and only if $m q>n$.

The fact that $m q>n$ implies the density property is easy to prove (see $\$ 2$ below); thus the interesting part of Theorem 1 lies in the construction of counterexamples whenever $m q \leqq n$. This is done in [6], [7] by a very explicit construction requiring detailed estimates (part of the complication there is due to the fact that the problem is formulated to include nonintegral values of $m$, an aspect which we ignore here). The purpose of the present paper is to show that a generalization of Theorem 1 may be proved with a noncomputational argument, by modifying the techniques used by Wermer and one of the present authors [2] in analyzing the operator $P(D)=\bar{\partial}$ in $R^{2}$.

For any point $\xi \in K$ we denote by $M_{K}(\xi)$ the norm of evaluation at $\xi$ on the space $H(K)$ :

$$
M_{K}(\xi)=\sup \left\{|u(\xi)|: u \in H(K),\|u\|_{L_{p}(K)} \leqq 1\right\} .
$$

Received by the editors September 21, 1972 and, in revised form, February 9, 1973. AMS (MOS) subject classifications (1970). Primary 35J30, 47F05.

Key words and phrases. Elliptic partial differential operator, bounded point evaluation.

${ }^{1}$ This work was supported in part by National Science Foundation Grants GP 33547 and GP 34625 .

(c) American Mathematical Society 1973 
Thus $0 \leqq M_{K}(\xi) \leqq \infty$. Our generalization of Theorem 1 may then be stated as follows.

THEOREM 2. If $m q \leqq n$, there exist a finite constant $C>0$ and two compact nowhere dense sets $K_{i} \subset \boldsymbol{R}^{n}$ having positive Lebesgue measure $(i=1,2)$ such that $K_{1} \subset K_{2}$ and for every $\xi \in K_{1}$ we have $M_{K_{2}}(\xi) \leqq C$.

If $K=K_{2}$ is the set constructed in Theorem 2, then $H(K)$ cannot be dense in $L_{p}(K)$, for this would imply that every function $f \in L_{p}(K)$ is bounded on $K_{1}$. We do not know whether the existence of a single point $\xi \in K$ with $M_{K}(\xi)<\infty$ implies that $H(K)$ is not dense in $L_{p}(K)$. Moreover, we do not know whether the compact sets $K$ such that $H(K)$ is dense in $L_{p}(K)$ can be characterized in terms of bounded $L_{p}$-evaluations, as was done in [2] for the case of the operator $P(D)=\bar{\partial}$ when $p \neq 2$.

2. Bounded point evaluations. We begin by recalling some elementary facts about an elliptic operator $P(D)$ acting on an open set $\Omega \subset \boldsymbol{R}^{n}$. We denote by $E$ any fundamental solution for $P(D)$ so that $E$ is a distribution satisfying $P(D) E=\delta$. We remark that the fundamental solution can be used to prove the easy part of Theorem 1: if $F \in L_{q}(K)$ annihilates $H(K)$, then $E * F$ is identically zero off $K$; since $P(D)(E * F)=F \in L_{q}$ and $P(D)$ is elliptic, we conclude [8, Theorem 7] that $E * F$ lies in the Sobolev space $W_{q}^{m}$ and hence is continuous in $\boldsymbol{R}^{n}$; thus $E * F \equiv 0$, so $F \equiv 0$.

If $H_{p}(\Omega)$ denotes the space of all functions $u \in L_{p}(\Omega) \cap C^{\infty}(\Omega)$ such that $P(D) u=0$ on $\Omega$, then $H_{p}(\Omega)$ is a closed subspace of $L_{p}(\Omega)$; indeed, any limit $u \in L_{p}(\Omega)$ of a sequence of functions $u_{n} \in H_{p}(\Omega)$ is obviously a weak solution of $P(D) u=0$, and hence, by the ellipticity of $P(D)$, a $C^{\infty}$ solution. Moreover, for any fixed point $\xi \in \Omega$ the mapping

$$
H_{p}(\Omega) \ni u \rightarrow u(\xi) \in C
$$

is a continuous linear functional. To prove this, we use the fact that $E$ must be of class $C^{\infty}$ on $\boldsymbol{R}^{n}-\{0\}$ by the ellipticity of $P(D)$. We also recall the easily verified fact that if $\alpha \in C_{0}^{\infty}$ is a function with support in a bounded neighborhood of the origin satisfying $\alpha \equiv 1$ on a smaller neighborhood of the origin, and $\operatorname{diam}[\operatorname{supp} \alpha]<\operatorname{dist}(\xi, \partial \Omega)$, then we have $P(D)[(1-\alpha) E]$ * $u \equiv u$ in some fixed neighborhood of $\xi$ for any $u \in H_{p}(\Omega)$ (see $[9, \S 3.1 .2]$ ). Since this represents $u(\xi)$ as an integral average of $u$ over a ball in $\Omega$, with a $C_{0}^{\infty}$ weight function, we conclude that (1) is a bounded mapping.

We denote by $M_{\Omega}(\xi)$ the norm of the mapping (1):

$$
M_{\Omega}(\xi)=\sup \left\{|u(\xi)| /\|u\|_{L_{p}(\Omega)}: u \in H_{p}(\Omega), u \neq 0\right\} .
$$

It is obvious that $M_{\Omega}(\xi)$ depends monotonically on the domain $\Omega$ : if $\xi \in \Omega_{1} \subset \Omega_{2}$ then $M_{\Omega_{2}}(\xi) \leqq M_{\Omega_{1}}(\xi)$. 
Now let $\Omega$ be a bounded domain in $\boldsymbol{R}^{n}$, and let $a$ and $\xi$ be two distinct points in $\Omega$. We let $B_{r}(a)$ denote the open ball of radius $r$ about the point $a$, and we define $\Omega_{r}=\Omega-\operatorname{cl}\left(B_{r}(a)\right)$. We wish to show that $M_{r}=M_{\Omega_{r}}(\xi)$ approaches $M_{\Omega 2}(\xi)$ as $r$ approaches 0 . From the monotonicity property we see that $\lim _{r \rightarrow 0} M_{r} \geqq M_{\Omega}(\xi)$. For each $r$ we may find a function $u_{r} \in$ $H_{p}\left(\Omega_{r}\right)$ such that $u_{r}(\xi)=1$ and $M_{r}-\left\|u_{r}\right\|_{L_{p}\left(\Omega_{r}\right)}^{-1}<r$. The function $u_{r}$ may be defined to be zero outside $\Omega_{r}$, and we then obtain a set of functions bounded in $L_{p}\left(\boldsymbol{R}^{n}\right)$, so for an appropriate sequence of indices $r \rightarrow 0$ the functions $u_{r}$ converge weakly in $L_{p}\left(\boldsymbol{R}^{n}\right)$ to a limit function $U$. Then for every $\phi \in C_{0}^{\infty}(\Omega-\{a\})$ we have, for sufficiently small $r, 0=\left\langle u_{r},{ }^{t} P(D) \phi\right\rangle \rightarrow$ $\left\langle U,{ }^{t} P(D) \phi\right\rangle$. Thus $P(D) U=0$ in $\Omega-\{a\}$. Since the hypothesis $m q \leqq n$ guarantees that isolated points are removable singularities for $P(D)$ (see [4, Theorem 1], [1], [5, Theorem 20]), we conclude that $P(D) U=0$ in $\Omega$. In view of the boundedness of the mapping (1) we see that $U(\xi)=1$, and hence

$$
M_{\Omega}(\xi)=\sup _{u \in H_{p}(\Omega) ; u \neq 0} \frac{|u(\xi)|}{\|u\|_{L_{p}(\Omega)}} \geqq \frac{1}{\|U\|_{L_{p}(\Omega)}} .
$$

If $\varepsilon>0$ is arbitrary, there exists a radius $R$ such that

$$
\|U\|_{L_{p}\left(\Omega_{R}\right)} \geqq\|U\|_{L_{p}(\Omega)}-\varepsilon \geqq 1 / M_{\Omega}(\xi)-\varepsilon .
$$

Since $u_{r} \rightarrow U$ weakly in $L_{p}\left(\Omega_{R}\right)$, we conclude that

$$
\begin{aligned}
1 / M_{\Omega}(\xi)-\varepsilon & \leqq\|U\|_{L_{p}\left(\Omega_{R}\right)} \\
& \leqq \lim \inf \left\|u_{r}\right\|_{L_{p}\left(\Omega_{R}\right)} \leqq \lim \inf \left\|u_{r}\right\|_{L_{p}\left(\Omega_{r}\right)}=1 / \lim M_{r} .
\end{aligned}
$$

This proves that $\lim _{r \rightarrow 0} M_{r} \leqq M_{\Omega}(\xi)$, and hence that $\lim _{r \rightarrow 0} M_{r}=M_{\Omega}(\xi)$.

We next prove that for a fixed domain $\Omega$, the function

$$
\Omega \ni \xi \rightarrow M_{\Omega}(\xi) \in \boldsymbol{R}
$$

is continuous. For this purpose we fix any point $\xi_{0} \in \Omega$, and we will find a neighborhood of $\xi_{0}$ on which this function is uniformly continuous. Let $d_{0}=\operatorname{dist}\left(\xi_{0}, \partial \Omega\right)$ and fix a function $\alpha \in C_{0}^{\infty}$ satisfying $\alpha=1$ near the origin and $\operatorname{diam}[\operatorname{supp} \alpha]<\frac{1}{2} \cdot d_{0}$. If $W=P(D)[(1-\alpha) E]$, we know that for all points $\xi \in B_{d_{0} / 2}\left(\xi_{0}\right)$ we may write $u(\xi)=\int u(t) W(\xi-t) d t$. We conclude that for points $\xi_{1}, \xi_{2} \in B_{d_{0} / 2}\left(\xi_{0}\right)$ we have

$$
\begin{aligned}
\left|u\left(\xi_{1}\right)-u\left(\xi_{2}\right)\right| & =\left|\int u(t)\left[W\left(\xi_{1}-t\right)-W\left(\xi_{2}-t\right)\right] d t\right| \\
& \leqq C_{0}\|u\|_{L_{p}(\Omega)} \omega_{W}\left(\left|\xi_{1}-\xi_{.2}\right|\right),
\end{aligned}
$$

where $C_{0}$ is the $L_{q}$ norm of the characteristic function of the ball $B_{d_{0}}\left(\xi_{0}\right)$, and $\omega_{W}$ denotes the modulus of continuity of the function $W$. Recalling 
the definition of $M_{\Omega}(\xi)$ we see that for points $\xi_{1}, \xi_{2} \in B_{d_{0} / 2}\left(\xi_{0}\right)$ we have

$$
\begin{aligned}
M_{\Omega}\left(\xi_{1}\right)-C_{0} \omega_{W}\left(\left|\xi_{1}-\xi_{2}\right|\right) & \leqq M_{\Omega 2}\left(\xi_{2}\right) \\
& \leqq M_{\Omega}\left(\xi_{1}\right)+C_{0}(1) \cdot\left(\left|\xi_{1}-\xi_{2}\right|\right) .
\end{aligned}
$$

This proves the continuity of the function (2).

3. Proof of Theorem 2. Let $K_{1}$ be a compact arc of positive Lebesgue measure contained in the open unit ball $B_{1}(0)$. If $\Omega$ is any domain containing $K_{1}$ we let $M_{\Omega}$ denote the maximum of the norms of evaluation at points of $K_{1}$ :

$$
M_{\Omega}=\max \left\{M_{\Omega}(\xi): \xi \in K_{1}\right\} .
$$

We now define a sequence of domains $\Omega_{j}$ inductively. We begin with $\Omega_{0}=B_{1}(0)$, and we let $\left\{b_{i}\right\}_{i=1}^{\infty}$ be an enumeration of all the points in $\Omega_{0}-K_{1}$ with rational coordinates. Let $a_{1}=b_{1}$; according to the results given at the end of the last section, together with Dini's theorem, we may find a region $\Omega_{1}=\Omega_{0}-\operatorname{cl}\left(B_{r_{1}}\left(a_{1}\right)\right)$ containing $K_{1}$ such that $M_{\Omega_{1}}$ differs from $M_{\Omega_{0}}$ by no more than $\frac{1}{2}$. Now suppose that the region $\Omega_{j-1}$ has been defined, and let $a_{j}$ be the first element of the sequence $\left\{b_{i}\right\}_{i=1}^{\infty}$ which lies in $\Omega_{j-1}$; again applying the results of the last section and Dini's theorem, we may find a region $\Omega_{j}=\Omega_{j-1}-\operatorname{cl}\left(B_{r_{i}}\left(a_{j}\right)\right)$ containing $K_{1}$ such that $M_{\Omega_{j}}$ differs from $M_{\Omega_{j-1}}$ by no more than $1 / 2^{j}$. The intersection

$$
K_{2}=\bigcap_{j=0}^{\infty} \operatorname{cl}\left(\Omega_{j}\right)
$$

is a compact set without interior, and $K_{1} \subset K_{2}$.

To complete the proof we must find a finite upper bound for the numbers $M_{K_{2}}(\xi)$ when $\xi \in K_{1}$. To do this consider an arbitrary function $u \in H\left(K_{2}\right)$. There exists an index $J$ such that $u \in H_{p}\left(\Omega_{j}\right)$ for all $j \geqq J$, and thus for each $\xi \in K_{1}$, we have

$$
|u(\xi)| \leqq M_{\Omega_{j}}\|u\|_{L_{p}\left(\Omega_{j}\right)} \leqq\left(M_{\Omega_{0}}+\sum_{j=1}^{\infty} \frac{1}{2^{j}}\right)\|u\|_{L_{p}\left(\mathrm{c} 1\left(\Omega_{j}\right)\right)}, \quad j \geqq J .
$$

By the Lebesgue monotone convergence theorem we see that $|u(\xi)| \leqq$ $\left(M_{\Omega_{0}}+1\right)\|u\|_{L_{p}\left(K_{2}\right)}$, and the proof is complete.

\section{REFERENCES}

1. D. R. Adams and J. C. Polking, The equivalence of two definitions of capacity, Proc. Amer. Math. Soc. 37 (1973), 529-534.

2. J. E. Brennan, Invariant subspaces and rational approximation, J. Functional Analysis 7 (1971), 285-310.

3. L. Hörmander, Linear partial differential operators, Die Grundlehren der math. Wissenschaften, Band 116, Academic Press, New York; Springer-Verlag, Berlin, 1963. MR 28 \#4221. 
4. W. Littman, Polar sets and removable singularities of partial differential equations, Ark. Mat. 7 (1967), 1-9. MR 37 \#559.

5. N. G. Meyers, A theory of capacities for potentials of functions in Lebesgue classes, Math. Scand. 26 (1970), 255-292. MR 43 \#3474.

6. J. C. Polking, A Leibniz formula for some differentiation operators of fractional order, Indiana Univ. Math. J. 21 (1972), 1019-1029.

7. - Approximation in $L^{p}$ by solutions of elliptic partial differential equations, Amer. J. Math. 94 (1972), 1231-1244.

8. R. T. Seeley, Refinement of the functional calculus of Calderón and Zygmund, Nederl. Akad. Wetensch. Proc. Ser. A 68=Indag. Math. 27 (1965), 521-531. MR 37 \#2040.

9. G. E. Šilov, Mathematical analysis: Second special course, "Nauka", Moscow, 1965; English transl., Generalized functions and partial differential equations, Gordon and Breach, New York, 1968. MR 36 \#2943; 37 \#5694.

Department of Mathematics, Indiana University, Bloomington, Indiana 47401

Department of Mathematics, University of Kentucky, Lexington, Kentucky 40506 